\title{
The design and achievement of interactive class response system
}

\author{
Zhao Mengwei ${ }^{1, a}$, Lu Qin ${ }^{2, b}$, Luo Wusheng ${ }^{3, c}$ \\ ${ }^{1,2,3}$ College of Mechatronics Engineering and Automation, National Univ. of Defense Technology, \\ Changsha 410073, China \\ a461158297@qq.com, bfreda0126@sina.com, 'Iwsheng0701@sina.com
}

Keywords: Visual Studio 2008, VSTO, ICRS, database

\begin{abstract}
Response information obtaining is a significant part in control system. Based on the modern information technology and control theory, ICRS (interactive class response system) can timely obtain available response information, then regulate and control teaching system. VSTO (Visual Studio Tools for the Microsoft Office System) provides many Windows form controls and creats user-defined task form for Office documents. This article is based on Visual Studio 2008 platform, VSTO technology and SQL database technology to design and research the software and hardware of ICRS. Server corresponds with the client through campus network (CN). Users connect the server interface with database by using VSTO technology, and manage the client received data. Meanwhile, teachers and students can see the real time response result on PowerPoint to adjust class content and teaching method in time, thus can improve the class teaching quality.
\end{abstract}

\section{Introduction}

In traditional class response teaching, the information response is often unmeasured and passive. And the feedback based on test has the problems of long feedback loop、 low working efficiency and so on, which greatly restrict the healthy development of education. Obtaining the response information timely and fully is difficult. ICRS is born to solve this problem [1].

Interactive class response system provides response information of all students to teacher timely, by adding ICRS equipments (sender and receiver) and relevant software solution. This article does research on the ICRS based on PC and smart hand-held terminal [2], Visual Studio platform and VSTO technology. This system can enhance the interaction between teachers and students, and improve the teaching efficiency and quality. ICRS is very suitable for professional curriculum interactive response teaching in universities.

\section{- Interactive class response system based on computer and smart hand-held terminal}

The hardware of the interactive class response system is shown in Figure 1. Teacher's personal computer connects campus network AP through WIFI module, as the server of campus network. Students send information directly to the teacher's server by using smart hand-held terminal. The server is connected with projector to show the response information on the screen. 


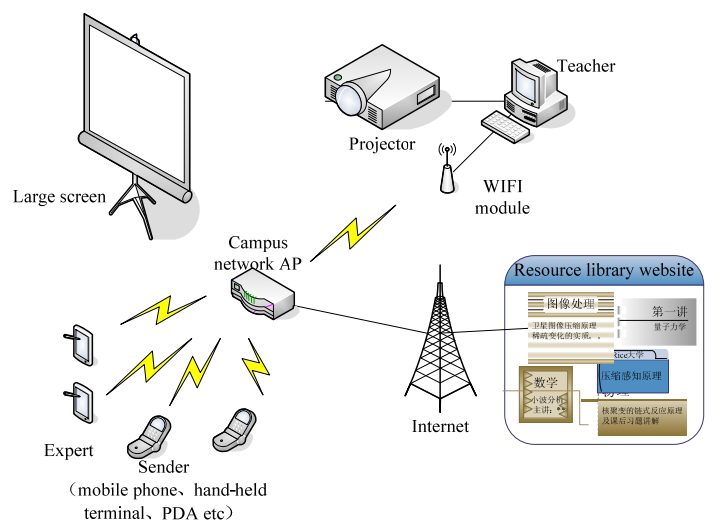

\section{二. The design of server system}

Figure.1 Interactive class response system

The hardware of the server includes PC(desktop computer)、projector、screen、reticle、power source、mouse and keyboard. Teacher and educational administration use computer connected to the campus network to receive the response information and timely project it to the screen.

The software has the following features:

(1) VSTO is based on Visual Studio platform, and the programming language is C\#.

VSTO combines Microsoft Office Professional with Microsoft Visual Studio.NET [3]. When teacher uses PowerPoint 2007, he can use application program by means of add-in to add response into class teaching. VSTO is mainly used to joint application program and Office element, as shown in Fig.2.

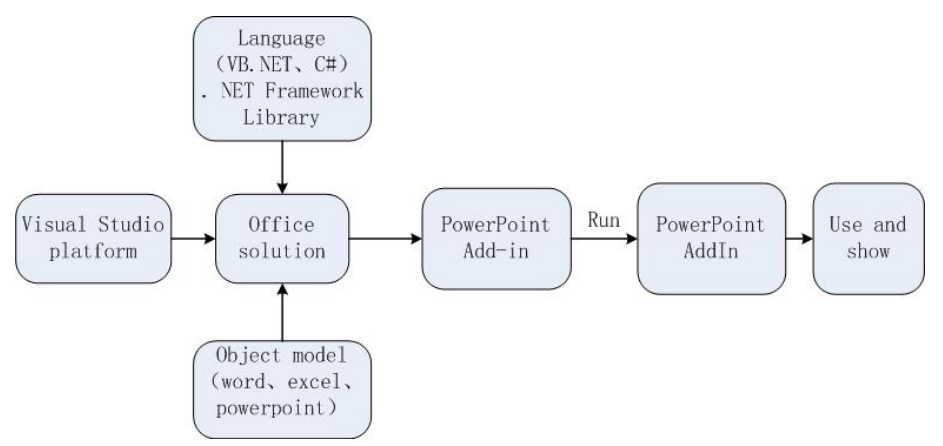

Figure 2 VSTO model

(2) Server has five function modules: hardware set、class response、vote and answer、data management and system set.

In the hardware set module, Http is choosn as the communication mode. As long as the smart hand-held terminal reaches the available range of campus network, the terminals will connect the server, and form the number-status table in the server so that teacher can check the on-line students at any times. Teachers can set the storage position of the response information through the system set module, other modules are used for the collection and management of class response information.

(3) To ensure system run fast and available, the software adopts multithread technology.

When initialize software, we create data receiving thread and user interface thread. The data receiving thread receives data from different clients by using cycling detection technology. As the data management is always behind the data receiving process, data receiving thread is also used for data handling. User interface thread is mainly used for user's operation.

(4) Adopt SQL database and establish sharing colleges and universities background database as support. 
When server initializes SQL database, department information table、 teacher information table、 course information table and other tables are created as well[4]. When receiving the response information, server updates the relevant fields in the database and forms new data table. Teachers are able to store the response information in the database, and they can also download relevant information from the database and resource library to distribute it to clients.

\section{三. The design of smart hand-held terminal system}

The main hardware of smart hand-held terminal is Tiny210V2 development board, along with camera module and WIFI module. The core board of Tiny210V2 is high-performance Cortex-A8, and Sumsung S5PV210 is the host processor. Tiny210V2 is shown in Figure 3.

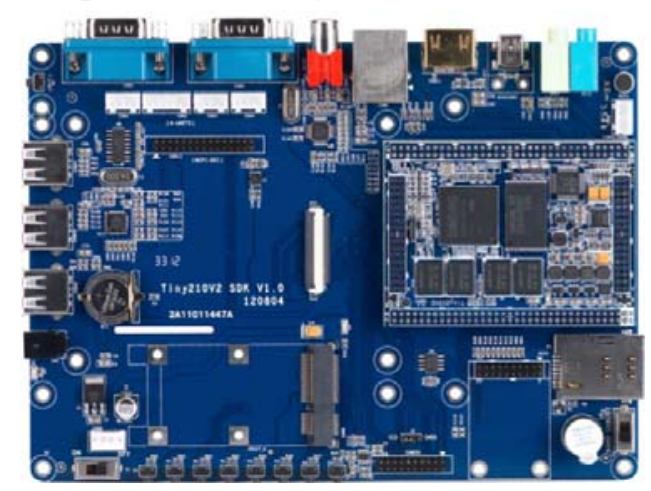

Figure 3 Tiny210V2 development board

The development board has one standard SD card seat in which SD card can be inserted to input and store pictures and data. CMOS camera can preview and take photos. When students want to sign in, terminals will send their photos which are named with date-time-number to server.

The hardware of terminal includes camera module、WIFI module、 data storage module and show module and so on. WIFI module is responsible for corresponding with the server. Camera module and data storage module will take photos and store data. The show module and audio output module is to output data and show it. The modules are shown in Figure 4.

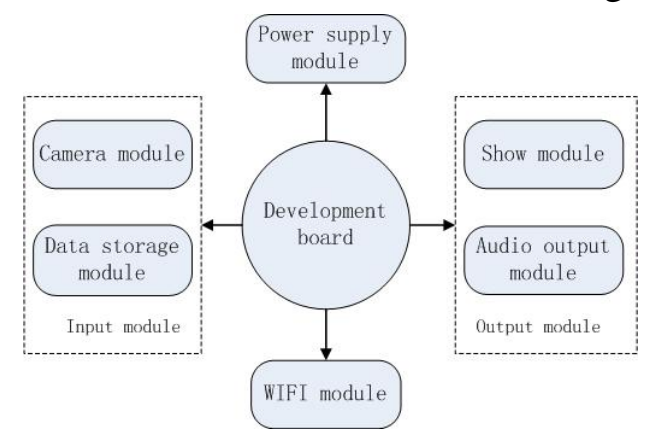

Figure 4 The hardware modules of client

In the software development process, we use Eclipse software as the platform and Java as the programming language. Besides, terminals adopt double thread technique, one is for background data management. The other is for user interface which is responsible for user's operation. 


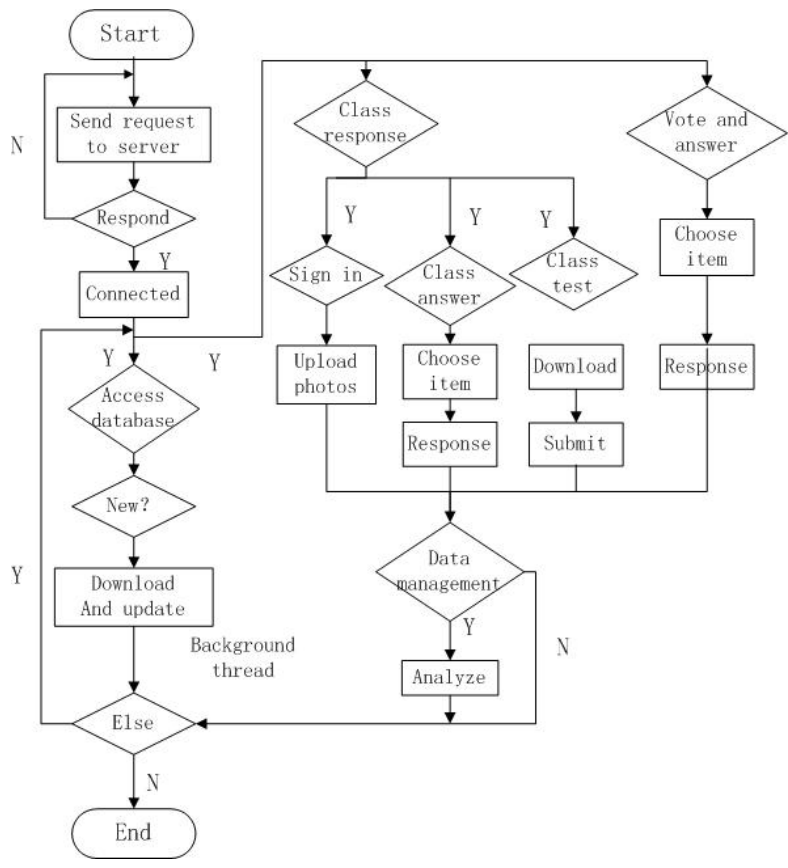

Figure 5 Client flowsheet

Http is chosen as the main corresponding method. Clients can communicate with campus network AP in the available range (about 100 meters) through WIFI. AP connects the host server using wired network, in accordance with IEEE802.11b corresponding rule, as is shown in Figure 6.

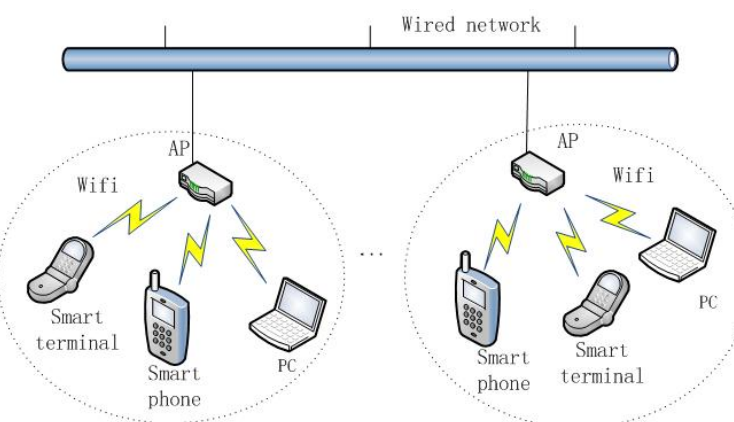

Figure 6 Network structure

Android client store data by android self-owned SQL database, and connected with server SQL database. The main data port adopts http webservice technology to visit server database directly, and the format of data returned is XML/JSON character string, predefined object type. Client can store the data in local database.

To insure the system run steadily at any time and clients can check relevant information off-line, when initializing, table structure and initialized data are created. When connected with server, clients download the relevant tables and data, and then store them in client database. In order to make sure the status of clients, server will detect the on-line clients at regular intervals. If the client does not answered for a period, server will stop sending message, considering this client as off-line. Normally, clients are always on-line.

\section{四. Conclusion}

The interactive class response system based on modern information technology is a new technology attempt which changed the traditional class response method. This article designed and studied the software and hardware of interactive class response system based on modern information technology. By using the PowerPoint 2007 add-in, we realized the server interface programming in class response so that users can directly use class response function when using 
PowerPoint 2007 [5]. SQL database can timely store response information and deal with relevant data. The use of multithread technology fully reflected the availability and real-time of class response. The achievement of this system uses the campus network and class equipments totally, with no additional cost, so it is very meaningful to popularize it.

\section{References}

[1] Douglas Slain. An Interactive Response System to Promote Active Learning in the Doctor of Pharmacy Curriculum[J]. American Journal of Pharmaceutical Education. Vol. 68(2004), p.117

[2] By Kathy Kenwright, Clickers in the Classroom, The Interdisciplinary Journal of Problem-based Learning, 53(1), (2009)

[3] Li Haohong, Using VSTO Technology to Extend Software Function of Office System. Fujian Computer, Vol. 6 (2010), p. 158-178

[4] Li Wei, Zhang Jiajie, Huang Hairui, Zhang Baorui. SQL Server Database Management and Application[C]. Tsinghua University Press. (2012), p. 2-10

[5]Yang Xueguang, Shi Huajie, Huang Liyong. Using VSTO to Update Excel Diagram[J]. 19 (2011), p. 154 